Open Access

\title{
The norpurpureine alkaloid from Annona purpurea inhibits human platelet activation in vitro
}

Gabriela Sánchez', Omar Estrada', Giovana Acha', Alfonso Cardozo², Franshelle Peña', Marie Christine Ruiz', Fabián Michelangeli ${ }^{1}$ and Claudia Alvarado-Castillo ${ }^{1,3^{*}}$ (i)

\begin{tabular}{l}
\hline * Correspondence: \\
cpilar.alvarado@gmail.com; \\
calvarad@ivic.gob.ve \\
${ }^{1}$ Centro de Biofísica y Bioquímica \\
(CBB), Instituto Venezolano de \\
Investigaciones Científicas (IVIC), \\
Caracas, Bolivarian Republic of \\
Venezuela \\
'Laboratorio de Hemostasia y \\
Genética Vascular, Centro de \\
Biofísica y Bioquímica, Instituto \\
Venezolano de Investigaciones \\
Científicas, Apartado 20632, K11 de \\
la Carretera Panamericana, Caracas \\
1020-A, Bolivarian Republic of \\
Venezuela \\
Full list of author information is \\
available at the end of the article
\end{tabular}

* Correspondence:

cpilar.alvarado@gmail.com;

calvarad@ivic.gob.ve

(CBB), Instituto Venezolano de Investigaciones Científicas (IVIC),

Caracas, Bolivarian Republic of

Venezuela

Genética Vascular, Centro de

Biofísica y Bioquímica, Instituto

Venezolano de Investigaciones

Científicas, Apartado 20632, K11 de

1020-A, Bolivarian Republic of

Full list of author information is

available at the end of the article

\begin{abstract}
Background: The leaves of Annona purpurea have yielded several alkaloids with anti-aggregation activities against rabbit platelets. This is promising in the search for agents that might act against platelets and reduce the incidence of cardiovascular diseases. Since significant differences in platelet function have been reported between human and animal platelets, a study focusing on the effect of $A$. purpurea extracts against human platelet activation is necessary.
\end{abstract}

Methods: The compounds in an A. purpurea ethanolic extract underwent bio-guided fractionation and were used for in vitro human platelet aggregation assays to isolate the compounds with anti-platelet activity. The bioactive compounds were identified by spectroscopic analysis. Additional platelet studies were performed to characterize their action as inhibitors of human platelet activation.

Results: The benzylisoquinoline alkaloid norpurpureine was identified as the major anti-platelet compound. The $\mathrm{IC}_{50}$ for norpurpureine was $80 \mu \mathrm{M}$ against platelets when stimulated with adenosine $5^{\prime}$-diphosphate (ADP), collagen and thrombin. It was pharmacologically effective from 20 to $220 \mu \mathrm{M}$. Norpurpureine $(220 \mu \mathrm{M})$ exhibited its in vitro effectiveness in samples from 30 healthy human donors who did not take any drugs during the 2 weeks prior to the collection. Norpurpureine also gradually inhibited granule secretion and adhesion of activated platelets to immobilized fibrinogen. At the intra-platelet level, norpurpureine prevented agonist-stimulated calcium mobilization and CAMP reduction. Structure-activity relationship analysis indicates that the lack of a methyl group at the nitrogen seems to be key in the ability of the compound to interact with its molecular target.

Conclusion: Norpurpureine displays a promising in vitro pharmacological profile as an inhibitor of human platelet activation. Its molecular target could be a common effector between $\mathrm{Ca}^{2+}$ and CAMP signaling, such as the PLC-PKC-Ca ${ }^{2+}$ pathway and PDEs. This needs further evaluation at the protein isoform level.

Keywords: Annona purpurea, Alkaloids, Norpurpureine, Human platelets

\section{Background}

Platelet activation is a key event in thrombus formation, chronic inflammation and atherosclerosis, which are all multicellular processes involved in the development of cardiovascular diseases. Currently, anti-platelet agents such as aspirin and clopidogrel

(c) The Author(s). 2018 Open Access This article is distributed under the terms of the Creative Commons Attribution 4.0 International License (http://creativecommons.org/licenses/by/4.0/), which permits unrestricted use, distribution, and reproduction in any medium, provided you give appropriate credit to the original author(s) and the source, provide a link to the Creative Commons license, and indicate if changes were made. The Creative Commons Public Domain Dedication waiver (http://creativecommons.org/ publicdomain/zero/1.0/) applies to the data made available in this article, unless otherwise stated. 
are widely used alone or in combination to reduce the incidence of ischemic stroke and to prevent arterial thrombosis [1]. Despite the efficacy of dual anti-aggregation therapy, growing evidence of drug resistance for aspirin and clopidogrel [2] highlight the need to search for novel anti-platelet agents to reduce the incidence of cardiovascular diseases; which are the globally leading causes of death and disability [3].

Various compounds with platelet anti-aggregation activity have been isolated from medicinal plants using animal platelets and in vitro bio-assays [4]. Several anti-platelet compounds have been identified from different species of the Annona genus (Annonaceae) using rabbit platelets: acid amines from $A$. montana [5], aporphine alkaloids from A. purpurea [6,7] and ent-kaurane diterpenoids from A. squamosa [8]. A. purpurea is widely distributed throughout the tropical and subtropical regions of Central America [9], making it an attractive source for pharmacologically active substances.

Significant differences in platelet function may exist between human and animal platelets [10-13], so these effects on rabbit platelets need to be assessed using a human model. In this study, we searched for anti-platelet compounds in the leaves of A. purpurea, using human platelets and a bio-guided fractionation of its ethanolic extract (EE). Additionally, we explored the pharmacological properties and mechanism of action of the isolated bioactive compounds.

\section{Methods}

Materials

Acetone, acetonitrile and acetic acid (reagent grade) were purchase from J.T. Baker Chemical. Methanol, ethanol, DMSO, ADP, ATP, thrombin, human fibrinogen, IBMX, PMA, probenecid, apyrase, cAMP enzyme immunoassay kit, Sephadex LH-20 and dimethylsulfoxide-d6 to NMR were from Sigma-Aldrich. Collagen was from Helena Laboratories. CHRONO-LUME was from Chrono-log Corporation and Fura-2-AM was from Invitrogen Corporation.

\section{Plant material}

The leaves of Annona purpurea Moc. \& Sessé ex Dunal [14] were collected in July 2008 at Parque Nacional Henri Pittier, Aragua, Venezuela. They were identified by Dr. Alfonzo Cardozo and a voucher specimen (AC27435) was deposited in the herbarium of Víctor Manuel Badillo (MY), Facultad de Agronomía, UCV, Maracay, Venezuela. The collection of plant material was done in accordance with the Biological Diversity Law of República Bolivariana de Venezuela (gaceta oficial número 5.468 extraordinario de fecha 24-05-2000), under permission number H-46 (date of issue: August 1, 2007; date of expiration: August 1, 2008). This was granted to the herbarium of Victor Manuel Badillo (MY) in the name of Alfonzo José Cardozo López and allows the collection of botanical samples for scientific research purposes.

\section{Blood collection and platelet preparations}

Blood was obtained by clean venipuncture from 30 healthy human donors who did not take any drugs during the 2 weeks prior to the collection. All donors gave informed consent for the study, which was approved by the Bioethical Committee of IVIC 
(number 1316, approval on March 2009), following the guidelines of the Declaration of Helsinki and Tokyo for humans.

Platelet-rich plasma (PRP) and washed platelets (WP) were obtained according to the method of Cazenave et al. [15], with modifications. Briefly, blood samples were collected, discarding the first 2 or $3 \mathrm{ml}$, into 3.2\% (109 mM) trisodium citrate didydrate (1:9 v/v, citrate to blood) and centrifuged at $160 \times \mathrm{g}$ for $15 \mathrm{~min}$ (with no brake), at room temperature (RT). PRP (the upper phase) was isolated and the remaining lower phase was further centrifuged at $1500 \times \mathrm{g}$ for $15 \mathrm{~min}$ at RT to obtain platelet-poor plasma (PPP), which is used to determine $100 \%$ light transmittance in platelet aggregation assays. WP were prepared from the PRP using blood anticoagulated with acid-citrate-dextrose (ACD) consisting of $38 \mathrm{mM}$ citric acid monohydrate, $85 \mathrm{mM}$ trisodium citrate dihydrate, and $123 \mathrm{mM}$ anhydrous $\mathrm{D}(+)$ glucose at pH 5 (1:6 v/v, ACD to blood). Then, PRP supplemented with $1 \mu \mathrm{M} \mathrm{PGE}_{1}$ was centrifuged at $1500 \times \mathrm{g}$ for $15 \mathrm{~min}$ at RT and the platelet pellet was washed once with HEPES-modified Tyrode buffer consisting of $134 \mathrm{mM} \mathrm{NaCl}, 2.9 \mathrm{mM} \mathrm{KCl}, 12 \mathrm{mM}$ $\mathrm{NaHCO}_{3}, 0.34 \mathrm{mM} \mathrm{NaH} \mathrm{PO}_{4}, 1 \mathrm{mM} \mathrm{MgCl}, 20 \mathrm{mM}$ HEPES (pH 6.5) and $5 \mathrm{mM}$ glucose; supplemented with $1 \mu \mathrm{M} \mathrm{PGE}_{1}$. The washed platelet pellet was carefully and slowly resuspended at 300,000 platelets/ $\mu \mathrm{l}$ in HEPES-modified Tyrode's buffer pH 7.4; supplemented with $0.35 \%$ albumin, $0.5 \mathrm{U} / \mathrm{ml}$ apyrase and $2 \mathrm{mM} \mathrm{CaCl}_{2}$, without $\mathrm{PGE}_{1}$. The resuspended platelets were rested at RT for at least $30 \mathrm{~min}$ and $0.25 \mathrm{mg} / \mathrm{ml}$ human fibrinogen was added prior to use.

\section{In vitro platelet aggregation assay}

Platelet aggregation was monitored using Born's turbidimetric method [16]. Inhibition experiments were done as described earlier [17] by incubating the platelets with different A. purpurea samples, norpurpureine and purpureine for $10 \mathrm{~min}$ before stimulation with the agonists: $10 \mu \mathrm{M}$ ADP, $1 \mu \mathrm{g} / \mathrm{ml}$ collagen (in PRP) and $0.075 \mathrm{U} / \mathrm{ml}$ thrombin (in WP). DMSO was used as a vehicle at a final concentration less than $0.25 \%$ in all the cases. Platelet aggregation responses were recorded for $10 \mathrm{~min}$ (Chrono-log 700 ), at $37^{\circ} \mathrm{C}$ with stirring at $1000 \mathrm{rpm}$.

\section{Bioassay-guided isolation and identification of anti-platelet compounds from A. purpurea leaves}

The anti-aggregation activities of the A. purpurea extract, fractions and isolated compounds against human platelets in vitro were tested at $250 \mu \mathrm{g} / \mathrm{ml}$. Only the active samples were then studied in the next phase of the separation process.

Powdered dried leaves $(300 \mathrm{~g})$ were subjected to percolation with ethanol for a week. The solvent was evaporated in vacuo to yield $52 \mathrm{~g}$ of dry ethanol extract (EE). Then, from the partition of $A$. purpurea EE in methanol-water (1:1) two fractions were obtained: a green residue (21.2 g) named methanol-water insoluble fraction (MWIF); and a red solution that after evaporation in vacuo yielded a red residue $(27.7 \mathrm{~g})$ named methanol-water soluble fraction (MWSF). MWSF was repeatedly extracted with acetone to obtain two new fractions: a brownish residue named acetone insoluble fraction (AIF); and a yellowish solution from which a yellowish residue named AF was yielded (11.7 g) after evaporation of the solvent. A portion of AF (2 g) was fractionated on Sephadex LH-20 column chromatography (CC) using methanol as the eluent to give three fractions, 
named I-III. From fraction II, two compounds: norpurpureine (A; $350 \mathrm{mg})$ and purpureine (B; $200 \mathrm{mg}$ ) were finally purified after CC on RP-18 using the mixture acetonitrile-wateracetic acid (65-30-0.5) as the eluent. The structures of compounds A and B were characterized in 1D and 2D NMR experiments and identified by comparison with spectroscopic data [18].

\section{Spectroscopic analysis}

${ }^{1} \mathrm{H}$ vand ${ }^{13} \mathrm{C}$ NMR spectra were performed in hexadeuterodimethylsulphoxide (DMSOd6) on a Brucker DRX 500 spectrometer at Centro de Química, IVIC. Mass spectra were measured in a Bruker Micro TOF-QIII spectrometer set to ESI mode using $\mathrm{MeOH}$ as the solvent at Centro de Biología Estructural, IVIC. NMR spectra of norpurpureine and purpureine (Additional file 1) and MS spectra of norpurpureine and purpureine (Additional file 2), are available.

\section{Measurements of ATP secreted from activated platelets}

The ATP released from platelets $\left(0.4 \mathrm{ml}\right.$ PRP adjusted to $\left.3.0 \times 10^{8} / \mathrm{ml}\right)$ was measured by adding $50 \mu \mathrm{l}$ of luciferin/luciferase reagent (CHRONO-LUME), $1 \mathrm{~min}$ before stimulation with $10 \mu \mathrm{M}$ ADP. Platelet aggregation and ATP secretion responses were simultaneously measured, at $37{ }^{\circ} \mathrm{C}$ with stirring at $1000 \mathrm{rpm}$, in a Lumi-aggregometer Model 700 (Chrono-Log Co.). The amount of ATP (nmols) was determined using ATP standard calibration.

\section{Adhesion assay of activated human platelets onto fixed fibrinogen}

Platelets $\left(1 \times 10^{7}\right)$ in PRP pre-treated with DMSO, apyrase or norpurpureine for $10 \mathrm{~min}$ (at the indicated concentrations) were stimulated with $10 \mu \mathrm{M}$ ADP for $3 \mathrm{~min}$ and then platelet adhesion onto fibrinogen-coated wells was allowed for $1 \mathrm{~h}$ at RT. The measurements of adherent platelets to fibrinogen were carried out according to Eriksson and Whiss [19].

\section{Quantification of cytosolic calcium concentrations}

Platelets in PRP supplemented with $2 \mathrm{mM}$ probenecid were incubated with the fluorescent calcium indicator Fura-2-AM $(5 \mu \mathrm{M})$ at $37{ }^{\circ} \mathrm{C}$ for $1 \mathrm{~h}$ in darkness. The washed pellet of Fura-2-AM-loaded platelets were resuspended with HEPES buffer supplemented with $2 \mathrm{mM}$ probenecid and $2 \mathrm{mM} \mathrm{CaCl}_{2}$, at a concentration of $1 \times 10^{9}$ platelets $/ \mathrm{ml}$. Platelets $(1 \mathrm{ml})$ were incubated with DMSO or norpurpureine for $10 \mathrm{~min}$ before addition of $0.075 \mathrm{U} / \mathrm{ml}$ thrombin. The measurements of $\left[\mathrm{Ca}^{2+}\right]_{\mathrm{i}}$ were performed at $37{ }^{\circ} \mathrm{C}$ in a MSIII fluorometer (Photon Technology International) equipped with a stirrer and temperature control, using alternate excitation wavelengths of 340 and $380 \mathrm{~nm}$ and an emission wavelength of $510 \mathrm{~nm}$ as previously described $[20]$. The $\left[\mathrm{Ca}^{2+}\right]_{\mathrm{i}}$ values were calculated using the SPEX dM3000 software package, according to the equation described by Grynkiewicz et al. [21].

\section{Measurements of cAMP levels in platelets}

Intra-platelet cAMP concentrations were measured using a commercially available kit. Briefly, platelets $\left(3 \times 10^{8} / \mathrm{ml}\right)$ were pre-incubated as indicated, for $10 \mathrm{~min}$ at $37^{\circ} \mathrm{C}$ with stirring at $1000 \mathrm{rpm}$, before agonist stimulation with $10 \mu \mathrm{M}$ ADP, $1 \mu \mathrm{g} / \mathrm{ml}$ collagen (in 
PRP) and $0.075 \mathrm{U} / \mathrm{ml}$ thrombin (in WP). The reaction was stopped by the addition of an equal volume of ice-cold ethanol; the samples were kept on ice for a further $45 \mathrm{~min}$ and centrifuged at $7000 \times \mathrm{g}$ for $15 \mathrm{~min}$ at $4{ }^{\circ} \mathrm{C}$. Each supernatant containing cAMP was evaporated to dryness in vacuo and reconstituted with water. cAMP levels were determined according to the manufacturer's specifications (cAMP Enzyme Immunoassay Kit, Sigma-Aldrich).

\section{Statistical analysis}

Values are expressed as means and standard deviations (SD). Statistical analysis was performed applying one-way ANOVA and Bonferroni post hot test using GraphPad Prism 6.1 software. Differences between responses were considered statistically significant at $\left.p<0.05(*), 0.01{ }^{(* *)}\right)$ and $0.001\left(^{* * * *}\right)$.

\section{Results}

Norpurpureine, the major anti-platelet compound isolated from $A$. purpurea leaves

We found that $A$. purpurea $\mathrm{EE}$ at $250 \mu \mathrm{g} / \mathrm{ml}$ exhibited modest anti-platelet aggregation effects against ADP, collagen and thrombin (between 30 and 45\%, n=5 per agonist). Bio-directed fractionation of this EE led to the isolation and identification of two known alkaloids: norpurpureine and purpureine (Fig. 1).

Interestingly, norpurpureine at $250 \mu \mathrm{M}$ ( $n=5$ per agonist) completely retained the anti-platelet effects observed for A. purpurea EE, while purpureine at $250 \mu \mathrm{M}$ ( $n=5$ per agonist) did not inhibit the aggregation of human platelets (Fig. 2). These findings indicate that norpurpureine is the major anti-platelet compound in A. purpurea leaves. Importantly, norpurpureine $(100 \mu \mathrm{g} / \mathrm{ml}$ for $30 \mathrm{~min}$, without agonists $)$ alters neither the number and morphology of platelets (40×, under light microscopy) nor the approximate number of platelet microparticles (mostly visible like bacteria at $100 \times$ ). This reveals that this alkaloid did not induce signs of platelet activation or toxicity by itself under the experimental conditions.

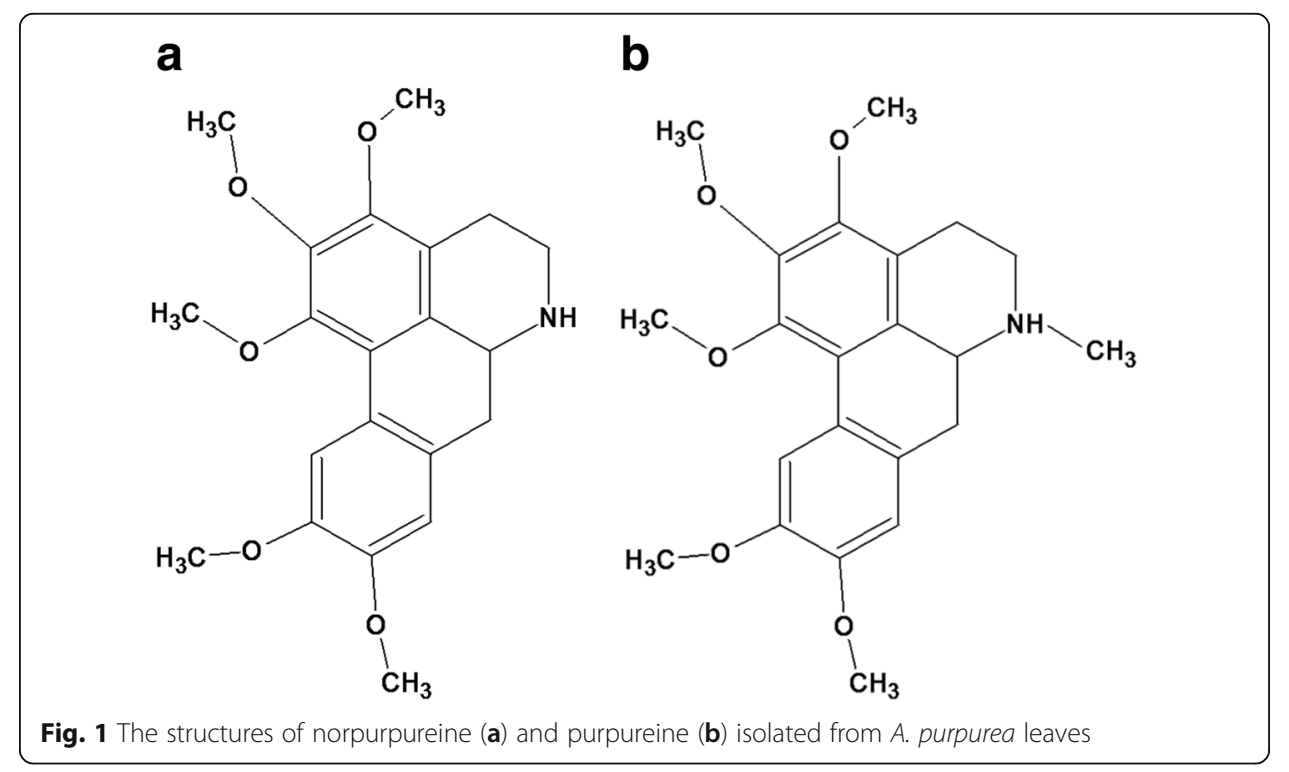




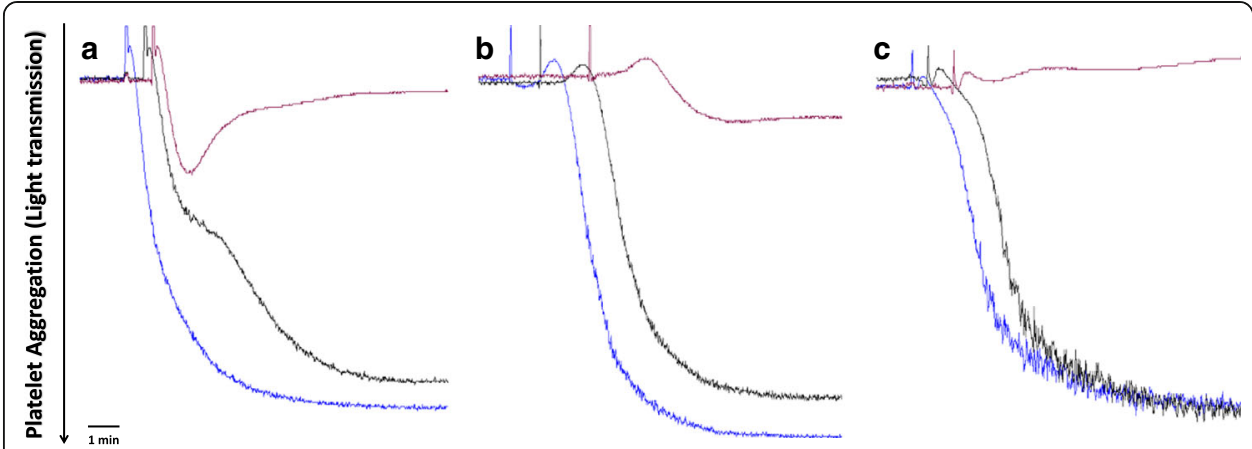

Fig. 2 Norpurpureine is a non-selective inhibitor of human platelet aggregation. Typical traces of platelet aggregation responses monitored by changes in the light transmission signal over time are shown. Platelets were incubated with the vehicle (0.25\% DMSO, blue), $250 \mu \mathrm{M}$ purpureine (black) or $250 \mu \mathrm{M}$ norpurpureine (red) for $10 \mathrm{~min}$ before their stimulation with $10 \mu \mathrm{M}$ ADP (a), $1 \mu \mathrm{g} / \mathrm{ml}$ collagen (b) (in PRP) or $0.075 \mathrm{U} / \mathrm{ml}$ thrombin (c) (in WP). These original tracings are representative of five curves done for each agonist

\section{Efficacy, potency and effectiveness of norpurpureine as inhibitor of human platelet activation}

Norpurpureine inhibited agonist-induced platelet aggregation in a concentration-dependent manner, showing a pharmacologically effective concentration range of $20 \mu \mathrm{M}\left[10^{\wedge}(-4.70)\right]$ to $220 \mu \mathrm{M}\left[10^{\wedge}(-3.65)\right]$, for each agonist: ADP, collagen and thrombin (Fig. 3). The Hill slopes of these three curves $(2.7 \pm 0.8$ for ADP; $7.53 \pm 2.9$ for collagen; and $3.9 \pm 1.9$ for thrombin; $p=0.056$ ) and the concentration values at which norpurpureine inhibits $50 \%$ of the maximal response $\left(\mathrm{IC}_{50}\right)(\mathrm{ADP}$ at $77.6 \pm 8 \mu \mathrm{M}$; collagen at $84.5 \pm 4 \mu \mathrm{M}$; and thrombin at $79.4 \pm 9 \mu \mathrm{M} ; p=0.44)$ are similar, indicating potency as anti-platelet agent around $80 \mu \mathrm{M}$ $(29.71 \mu \mathrm{g} / \mathrm{ml})$. It is remarkable that norpurpureine was pharmacologically effective (at $220 \mu \mathrm{M})$ in samples from 30 apparently healthy blood donors, providing evidence of its in vitro effectiveness as an anti-platelet agent.

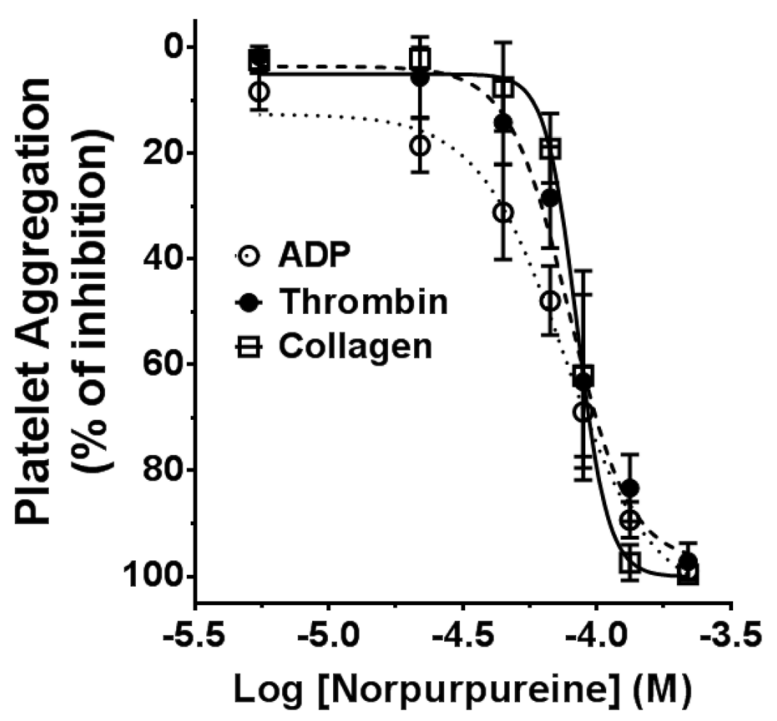

Fig. 3 Potency of norpurpureine as an anti-platelet agent. Concentration-response curves for norpurpureine inhibitory actions on agonist-induced platelet aggregations were constructed. Each data point, mean \pm SD $(n=5)$, is presented as the percentage reduction of the maximal amplitude response (versus the control, in the absence of norpurpureine). All measures were taken $10 \mathrm{~min}$ after the addition of the stimuli 


\section{Norpurpureine inhibits platelet secretion}

Since granule secretion is a common amplification event during platelet activation, we evaluated the ability of norpurpureine to modulate the amount of ATP released from dense granules of platelets activated by ADP (Fig. 4). Norpurpureine inhibited the agonist-induced secretory response in a concentration-dependent manner, exhibiting a significant inhibitory response near its $\mathrm{IC}_{50}$ value $(80 \mu \mathrm{M})$.

Norpurpureine prevents the adhesion of activated platelets to fibrinogen

As the formation of fibrinogen bridges between adjacent activated platelets is an important step for platelet aggregation, we evaluated whether norpurpureine affected the binding of fibrinogen to its receptor (activated integrin $\alpha \mathrm{IIb} \beta 3$ ) during platelet activation. In Table 1, norpurpureine gradually prevents the adhesion of ADPstimulated platelets onto fixed fibrinogen. This is similar to the effect of apyrase, a phosphatase that avoids platelet activation by dephosphorylation of ADP to AMP. These results suggest that norpurpureine inhibits the agonist-induced activation (insideout) of the integrin $\alpha \operatorname{IIb} \beta 3$, thereby preventing fibrinogen binding to activated platelets.

\section{Norpurpureine blocks the agonist-induced elevation of $\left[\mathrm{Ca}^{2+}\right]_{\mathrm{i}}$ in human platelets}

Given the critical role of $\left[\mathrm{Ca}^{2+}\right]_{\mathrm{i}}$ elevation during platelet activation [22], we investigated whether norpurpureine could modulate the $\left[\mathrm{Ca}^{2+}\right]_{\mathrm{i}}$ changes induced by thrombin on human platelets (Fig. 5). Norpurpureine prevents the agonist-stimulated elevation of $\left[\mathrm{Ca}^{2+}\right]_{\mathrm{i}}$, reducing the amplitude of the response in a concentration-

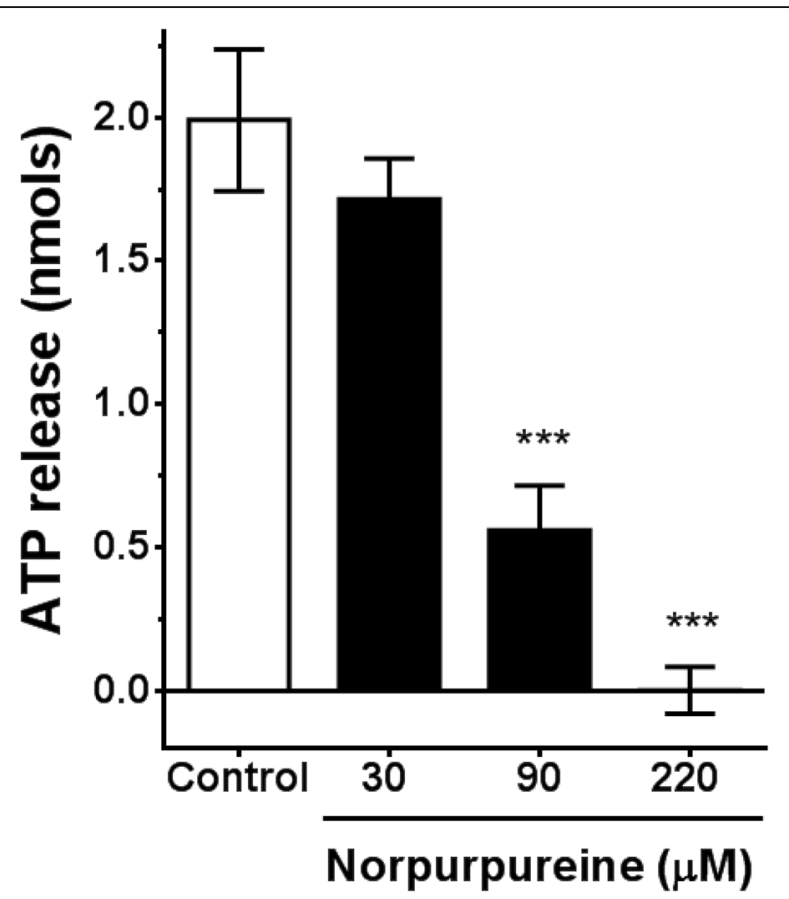

Fig. 4 Norpurpureine inhibits platelet secretion. Platelets were stimulated with $10 \mu \mathrm{M}$ ADP in the absence or presence of the indicated concentrations of norpurpureine. The amount of ATP released was quantified from the bioluminescence of the ATP-luciferin/luciferase reaction. The ATP standard calibration curve was used for

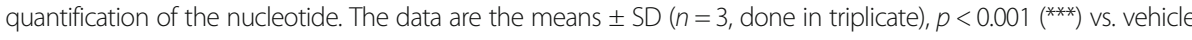


Table 1 Norpurpureine prevents ADP-induced platelet adhesion onto fixed fibrinogen

\begin{tabular}{|c|c|c|c|c|}
\hline & \multicolumn{2}{|c|}{ Net Adhesion (AU) } & \multirow{2}{*}{$\begin{array}{l}\text { Platelet-specific } \\
\text { adhesion (PSA) to Fg } \\
\text { induced by ADP }\end{array}$} & \multirow{2}{*}{$\begin{array}{l}\text { PSA (\%) vs. } \\
\text { vehicle }\end{array}$} \\
\hline & $\overline{A D P}(-)$ & $\operatorname{ADP}(+)$ & & \\
\hline Vehicle & $0.39(0.04)$ & $0.95(0.05)$ & $0.56(0.05)$ & $100.00(4.7)$ \\
\hline Norp $220 \mu \mathrm{M}$ & $0.42(0.03)$ & $0.51(0.04)$ & $0.09(0.01)$ & $16.07(1.8)^{* * *}$ \\
\hline Norp $90 \mu \mathrm{M}$ & $0.41(0.02)$ & $0.73(0.04)$ & $0.32(0.02)$ & $56.55(3.7)^{* * *}$ \\
\hline Norp $30 \mu \mathrm{M}$ & $0.42(0.03)$ & $0.85(0.02)$ & $0.43(0.03)$ & $76.78(4.7)^{* *}$ \\
\hline Apyrase & $0.40(0.02)$ & $0.49(0.03)$ & $0.09(0.02)$ & $16.67(2.7)^{* * *}$ \\
\hline
\end{tabular}

Platelets in PRP $\left(1 \times 10^{5}\right)$ were pretreated with vehicle (DMSO $\left.0.25 \%\right)$, apyrase $(0.5 \mathrm{U} / \mathrm{ml})$ or norpurpureine (Norp) at the indicated concentrations for $10 \mathrm{~min}$ before the addition of $10 \mu \mathrm{M}$ ADP to test their adhesion onto fixed fibrinogen (Fg). The values are the means \pm SD $\left(n=3\right.$, done in triplicate) $p<0.01\left(^{* *}\right)$ and $p<0.001{ }^{(* *)}$ compare to vehicle.

dependent manner (Fig. 5a). This inhibitory effect became significant from a low micromolar range $\sim 5 \mu \mathrm{M}$ (Fig. $5 \mathrm{~b}$ ) and was almost completely blocked around $80 \mu \mathrm{M}$ $\left(\mathrm{IC}_{50}\right)$.

\section{Protein kinase $C$ activation reversed the inhibitory action of norpurpureine on agonist-stimulated platelet aggregation}

Protein kinase $\mathrm{C}$ (PKC) is a common effector of signaling pathways triggered by the activation of different platelet receptors [23]. Therefore, we tested whether the anti-platelet effect of norpurpureine was affected by PKC activation (Fig. 6). We found that the phorbol ester PMA, a non-selective PKC activator, totally reversed the inhibition of norpurpureine on platelet aggregations stimulated by ADP and collagen but only partially reversed that inhibitory response on platelets stimulated by thrombin.

These results suggest that norpurpureine is an inhibitor of PKC activation or that its molecular target is upstream of PKC. This also reveals a complex regulation of PKC in human platelets, supporting evidence that platelet secretion and integrin activation could be positively or negatively regulated by different isoforms of PKC, which in turn may differ from one agonist to another [23].
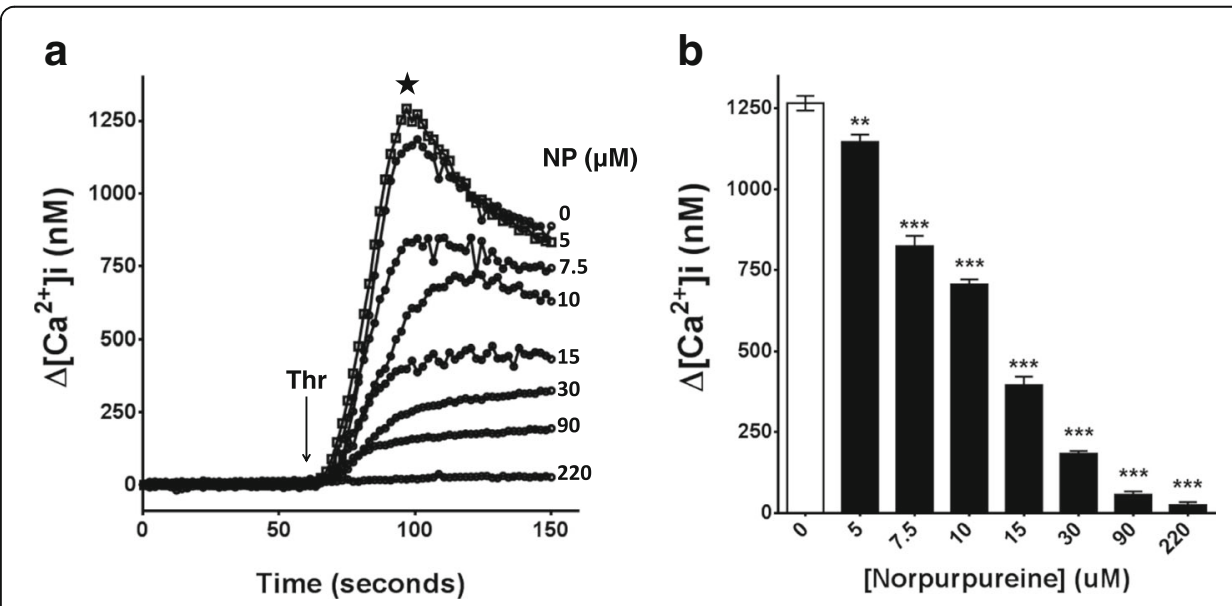

Fig. 5 Norpurpureine inhibits agonist-induced elevation of $\left[\mathrm{Ca}^{2+}\right]_{i}$ in human platelets. a Typical superimposed traces of $\left[\mathrm{Ca}^{2+}\right]_{i}$ changes stimulated by $0.075 \mathrm{U} / \mathrm{ml}$ thrombin (Thr) over time, in Fura-2-AM-loaded platelets pretreated with norpurpureine at the indicated concentrations. $\mathbf{b}$ Concentration-dependent inhibition of norpurpureine on the maximum elevation ( $\star$ in $5 \mathrm{~A}$, for each curve) reached by $\left[\mathrm{Ca}^{2+}\right]_{\mathrm{i}}$ after thrombin stimulation. Each data point is the mean $\left.\pm \mathrm{SD}(n=3), p<0.011^{* *}\right), p<0.001\left(^{* *}\right)$ vs. vehicle 


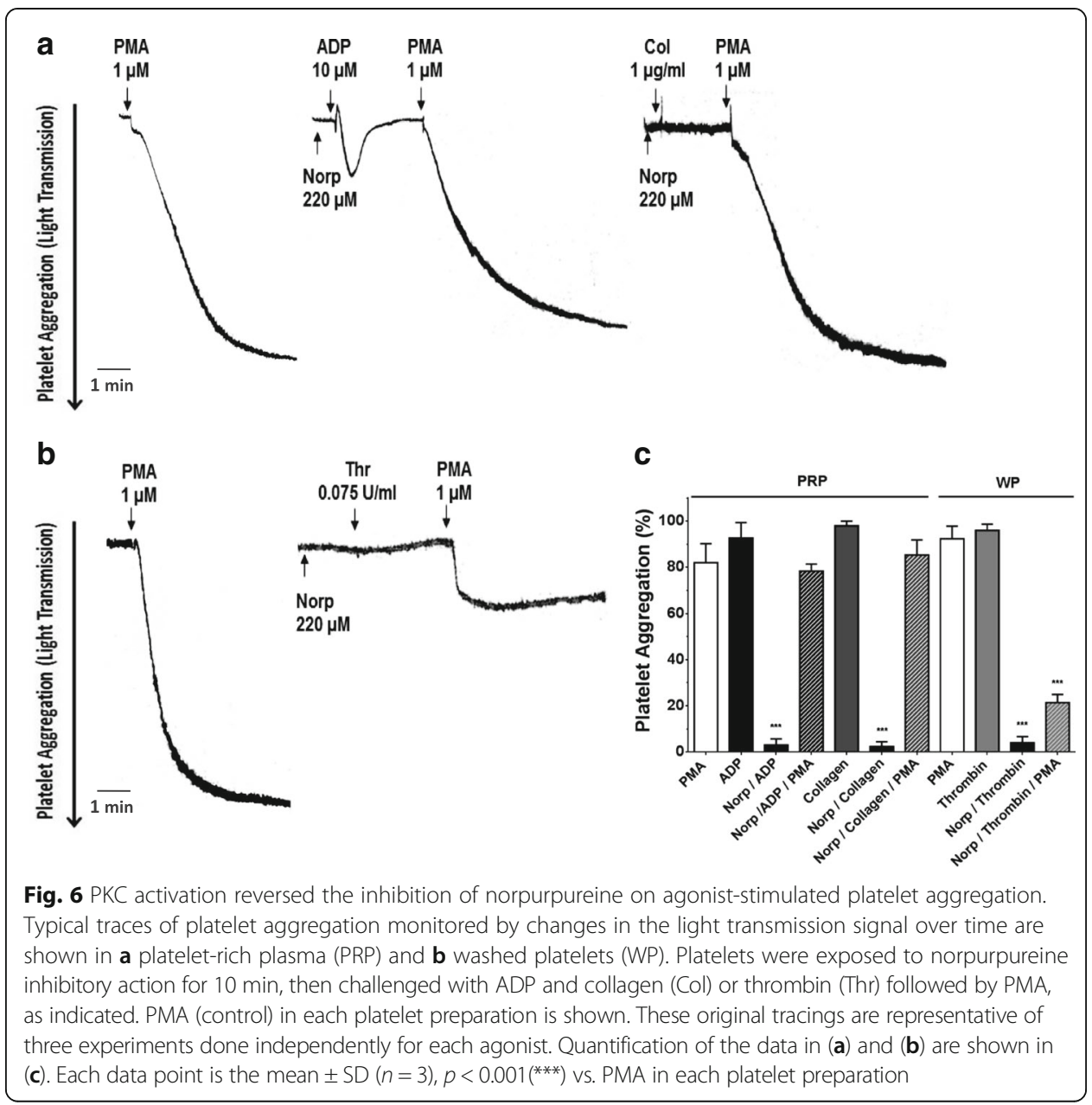

Norpurpureine prevents the agonist-induced decrease in cAMP levels in human platelets Activation of platelets involves the reduction of intracellular cAMP levels, mainly due to inhibition of adenylyl cyclases or activation of phosphodiesterases (PDEs), which are respectively enzymes that catalyze its synthesis and degradation [24]. Therefore, we investigated the effect of norpurpureine on the changes in intra-platelet cAMP levels after agonist-induced platelet activation. As shown in Fig. 7, norpurpureine and IBMX, a non-selected inhibitor of PDEs, did not modify the cAMP levels of resting platelets but significantly prevented the reductions in basal cAMP levels induced by collagen and thrombin. Similar results were obtained for ADP. Thus, the inhibitory action of norpurpureine involves prevention of cAMP degradation and it may be acting as an inhibitor of platelet PDEs.

\section{Discussion}

In this study, we used $A$. purpurea leaves collected in Maracay, Venezuela and human platelets. We found that the A. purpurea EE inhibited the aggregation of human platelets induced by ADP, collagen and thrombin; and that these anti-aggregating activities were 


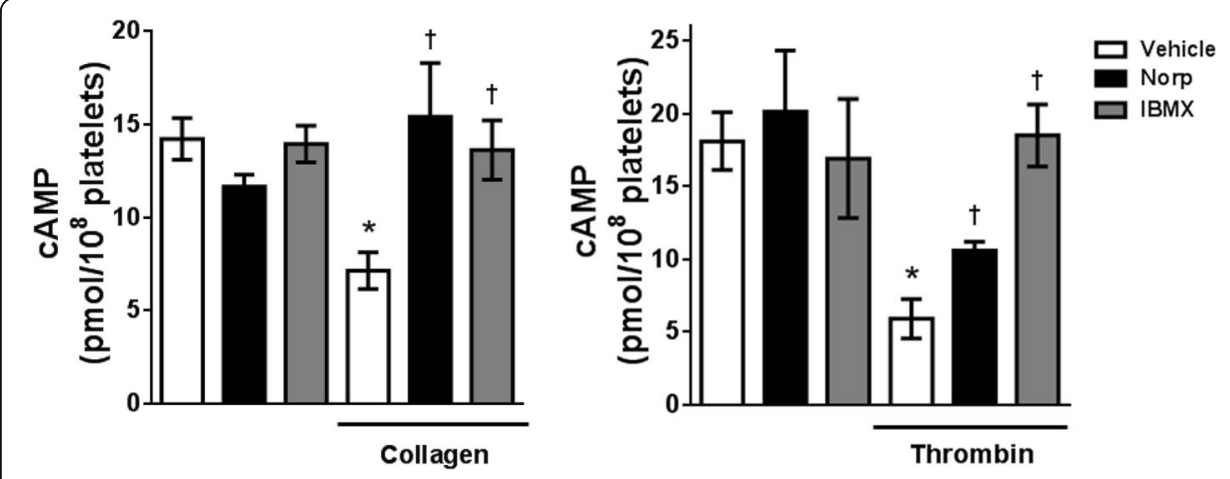

Fig. 7 Norpurpureine prevents the agonist-induced decrease in intra-platelet CAMP levels. Platelets were pre-treated for $10 \mathrm{~min}$ with vehicle (0.25\% DMSO), $220 \mu \mathrm{M}$ norpurpureine and $10 \mu \mathrm{M}$ IBMX; and stimulated with $1 \mathrm{\mu g} / \mathrm{ml}$ collagen (in platelet-rich plasma, PRP) and $0.075 \mathrm{U} / \mathrm{ml}$ thrombin (in washed platelets, WP). Data are the means \pm SD $\left(n=2\right.$, done in triplicate). $\left.p<0.05{ }^{*}\right)$ compare to basal (PRP or WP) and $p<0.05$ ( $\dagger$ ) compared to collagen- or thrombin-activated platelets

retained by the alkaloid fraction. Similar results were reported by Chang et al. [6, 7] for $A$. purpurea extract (leaves collected in Chia-Yi, Taiwan) using rabbit platelets. Those authors identified nine [6] and five [7] alkaloids with anti-platelet actions.

In this work, we identified two of the five known alkaloids isolated by Chang et al. [7]: norpurpureine and purpureine (thalicsimidine). Norpurpureine was found to be the major anti-platelet compound of A. purpurea leaves, showing activity against ADP, collagen and thrombin in human platelets. Purpureine did not inhibit human platelet activation.

Chang et al. [7] reported that $100 \mu \mathrm{g} / \mathrm{ml}(269.2 \mu \mathrm{M})$ norpurpureine completely inhibited the actions of arachidonic acid, collagen and platelet-activating factor (PAF) but only partially inhibited (30\%) the action of thrombin. They also found that $100 \mu \mathrm{g} / \mathrm{ml}$ $(259.4 \mu \mathrm{M})$ purpureine had variable inhibitory potency against arachidonic acid (85\%), collagen (63\%) and PAF (40\%) and no effect against thrombin in rabbit platelets. Thus far, it seems that norpurpureine (at $250 \mu \mathrm{M}$ ) is a nonselective inhibitor of human and rabbit platelets with better a platelet anti-aggregating profile than purpureine. It is noteworthy that purpureine inhibits rabbit platelets with greater effect than human platelets, suggesting that significant differences may exist between rabbit and human platelets at the level of its unknown molecular target.

The anti-platelet effects of norpurpureine and purpureine analyzed in terms of structureactivity relationships indicate the lack of a methyl group at the nitrogen in norpurpureine as the key feature by which these aporphine alkaloids interact with their molecular targets. This agrees with Chia et al. [25], who found that a small change in the structure of different sub-types of isoquinoline alkaloids caused significant changes in anti-platelet aggregation activity. On the other hand, by sharing the majority of their molecular structure, these alkaloids should also share most of their non-specific interactions, which makes the antiplatelet actions of norpurpureine less likely to be mediated by the induction of non-specific interactions in membrane fluidity, as suggested for several bioactive natural products [26].

As an anti-platelet agent, norpurpureine proved pharmacologically active from 20 to $220 \mu \mathrm{M}$, with a potency of $80 \mu \mathrm{M}$, and an $\mathrm{IC}_{50}$ value lower than that of aspirin 
$(140 \mu \mathrm{M})$ and ticlopide $(510 \mu \mathrm{M})$ obtained under similar in vitro conditions [27]. Importantly, norpurpureine was pharmacologically effective $(220 \mu \mathrm{M})$ in all 30 human platelet samples tested, which is evidence of its effectiveness and reveals that, at least $10 \mathrm{~min}$ prior to and during the $10 \mathrm{~min}$ of the aggregation response, it does not seem to be affected by the variability in oxidation and lipid state of these 30 PRP samples. Moreover, norpurpureine also gradually inhibited platelet granule secretion and adhesion of activated platelets to adhesive proteins like fibrinogen, suggesting that beyond hemostasis and thrombosis, this alkaloid could also modulate inflammatory and immunomodulatory activities, where these platelet functions have essential roles, particularly mediating intercellular communication [28].

Importantly, the cytotoxicity assessment of norpurpureine $(100 \mu \mathrm{g} / \mathrm{ml}$ for $48 \mathrm{~h})$ using the sulforhodamine B assay (available as Additional file 3) was promising. The compound reduced the initial cell populations of rhesus monkey kidney cell line MA104, human colon adenocarcinoma cell line HT29 and breast cancer mouse cell line 4 T1 by less than $10 \%$. Additionally, the cytotoxicity assessment of norpurpureine (for $72 \mathrm{~h}$ ) using alamar blue assay reports an $\mathrm{IC}_{50}$ value of $48.18 \mu \mathrm{M}$ for peripheral blood mononuclear cells (PBMCs) [29]. Thus, it is likely that the anti-platelet effects of norpurpureine, exerted in $10 \mathrm{~min}$, correspond to pharmacological rather than toxicological effects.

The three agonists used in this study act through different receptors and signal transduction mechanisms: ADP acts via Goq-mediated $\mathrm{P}_{2} \mathrm{Y}_{1}$ and Goi-mediated $\mathrm{P}_{2} \mathrm{Y}_{12}$ receptors; collagen acts mainly through tyrosine kinase-mediated immunoglobulin GP VI; and thrombin through $\mathrm{G} \alpha\left(\mathrm{q}, 12\right.$ and $\left.\mathrm{i}_{\mathrm{o}}\right)$-mediated $\mathrm{PAR}_{1}$ and $\mathrm{G} \alpha(\mathrm{q}, 12)$-mediated $\mathrm{PAR}_{4}$ receptors [30]. Activation of these receptors triggers different signaling pathways that converge into common signaling events to stimulate platelet shape change, granule secretion and aggregation to support platelet function. Thus, the observation that norpurpureine inhibits the actions of three different agonists with similar potency $\left(\mathrm{IC}_{50}\right.$ around $80 \mu \mathrm{M})$, strongly suggests that its molecular target should be a common downstream effector of the signaling pathways activated by these agonists.

Since norpurpureine gradually affected the amplitude of transient elevation in $\left[\mathrm{Ca}^{2+}\right]_{\mathrm{i}}$ induced by thrombin, its mechanism of action likely involves the negative regulation of the agonist-stimulated raise in $\left[\mathrm{Ca}^{2+}\right]_{\mathrm{i}}$. This correlates well with its potency to inhibit the second wave of platelet aggregation and granule secretion, and the adhesion of activated platelets to fibrinogen. In platelets, as in other non-excitable cells, increments in $\left[\mathrm{Ca}^{2+}\right]_{\mathrm{i}}$ involve the release of $\mathrm{Ca}^{2+}$ sequestered in the dense tubular system (DTS, the equivalent of the endoplasmic reticulum in platelets), followed by $\mathrm{Ca}^{2+}$ influx through the plasma membrane, a process referred as store-operated calcium entry (SOCE) [22]. Thus, norpurpureine actions probably involve the negative regulation of $\mathrm{Ca}^{2+}$ release from the DTS.

Activation of platelets by ADP and thrombin (G protein-coupled receptors) is via phospholipase $C$ beta (PLC $\beta$ ), while collagen (protein-tyrosine kinase receptor, GPVI) acts via $\operatorname{PLC} \gamma(2)$ [30]. PLC activation generates inositol 1,4,5-trisphosphate $\left(\mathrm{IP}_{3}\right)$ and diacylglycerol (DAG) from phosphatidylinositol 4,5-bisphosphate $\left(\mathrm{PIP}_{2}\right), \mathrm{IP}_{3}$ activates its receptors $\left(\mathrm{IP}_{3}-\mathrm{R}\right)$ on the DTS to release $\mathrm{Ca}^{2+}$ into cytosol. DAG, together with $\mathrm{Ca}^{2+}$, activates PKC allowing downstream PKC-dependent events that regulate different steps during platelet activation [23]. It is interesting that the PKC activator PMA, a DAG 
analog, fully rescued the aggregation response inhibited by norpurpureine in platelets stimulated by ADP and collagen but only partially rescued that response in platelets stimulated by thrombin. Human platelets express at least seven of the 12 PKC isoforms, namely conventional PKC $\alpha, P K C \beta I, P K C \beta I I$ (regulated by both DAG and $\mathrm{Ca}^{2+}$ ) and

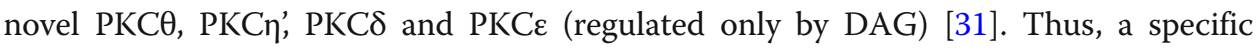
PKC isoform (or may be upstream of PKC, at the PLC level) could be the molecular target of norpurpureine. However, additional detailed studies will be required, since the specific PKC isoforms activated downstream of each receptor are not clearly understood and PKC play isoform-specific inhibitory and stimulatory roles in platelet activation [23].

Agonist-induced reduction in cAMP is a key signaling step to remove the negative regulation of cAMP-dependent protein kinase (PKA) on calcium-related signaling elements, such as PLC- $\beta 3$ [32] and $\mathrm{IP}_{3}$ receptors [33]. Under our experimental conditions, norpurpureine did not significantly modify intra-platelet cAMP in resting platelets, but significantly prevented the reduction in cAMP levels induced by the agonists used. Similar results were observed for IBMX, which strongly suggests the ability of norpurpureine to prevent the activation of PDEs in platelets. Human platelets express three PDE isoenzymes (PDE2, PDE3 and PDE5) and cAMP is hydrolyzed by PDE2 and PDE3 [34]. PDE3A is the most abundant isoform in platelets and has a 250-fold lower Km for cAMP than PDE2 [35]. Different platelet agonists, including thrombin, significantly enhance the activity of PDE3A in a phosphorylationdependent manner, actions that require the activation of PKC [36]. Further examination is needed to determine whether norpurpureine targets a PDE isoform to potentiate the negative regulation of cAMP on $\mathrm{Ca}^{2+}$ homeostasis or regulates cAMP levels via PKC.

Beyond platelets, anti-plasmodial activity [37] and in vitro cytotoxic activity toward the tumor cell lines [29] have been reported for norpurpureine. So far, no other types of biological activities have been reported for purpureine. Based on our results, in future studies it will be interesting to explore the effect of these alkaloids on the activity of different PLC, PKC and PDE isoforms in human and rabbit platelets, to have additional evidence on their structure-activity relationships and their molecular mechanisms as anti-platelet agents.

\section{Conclusions}

We have shown for the first time that the benzylisoquinoline alkaloid norpurpureine, unlike purpureine, acts as a non-selective inhibitor of human platelet activation. The in vitro pharmacological profile of norpurpureine as anti-platelet agent is: $\mathrm{IC}_{50}$ value of $80 \mu \mathrm{M}$ (potency); capacity to inhibit the action of three strong agonists of in vivo human platelet aggregation (efficacy); effective in at least 30 samples of platelets in plasma samples (PRP) from healthy donors (effectiveness). This in vitro pharmacological profile will help to support future studies of norpurpureine as an anti-thrombotic agent using animal models to establish its pharmacokinetic and pharmacodynamic profiles. Finally, we provide evidence that the molecular target of norpurpureine could be a common effector between $\mathrm{Ca}^{2+}$ and cAMP signaling, such as the PLC-PKC-Ca ${ }^{2+}$ pathway and PDEs. This needs further evaluation at the protein isoform level. 


\section{Additional files}

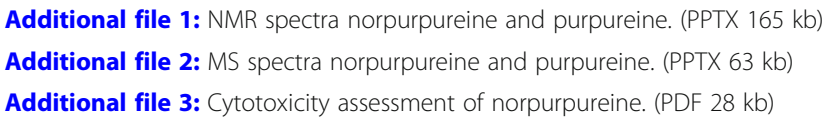

\section{Abbreviations}

ADP: Adenosine 5'-diphosphate; AMP: Adenosine 5'-monophosphate; ATP: Adenosine 5'-triphosphate; CAMP: Cyclic adenosine 3'-5'-monophosphate; DAG: Diacylglycerol; DMSO: Dimethyl sulfoxide; DTS: Dense tubular system; EE: Ethanolic extract; Fura-2-AM: Fura-2 acetoxymethyl ester; IBMX: 3-isobutyl-1-methylxanthine; $I C_{50}$ : Half maximal (50\%) inhibitory concentration; $I P_{3}$ : Inositol 1,4,5-trisphosphate; NMR: Nuclear magnetic resonance; PAF: Platelet activating factor; PDEs: Phosphodiesterases; PIP ${ }_{2}$ : Phosphatidylinositol 4,5-bisphosphate; PKA: CAMP-dependent protein kinase; PKC: Protein kinase C; PLC: Phospholipase C; PMA: Phorbol 12-myristate 13-acetate; PRP: Platelet-rich plasma

\section{Acknowledgments}

We would like thank IVIC's Dr. Sara Pekerar and Eva Vonaseck for their support with the spectroscopic experiments and B.S. Crisol Osorio for her technical assistance with the adhesion experiments.

\section{Funding}

This research was supported by IVIC (grants 1067 and 1227) and Fondo Nacional de Ciencia y Tecnología (FONACIT, grant Misión Ciencia 20071585), of the Bolivarian Republic of Venezuela.

\section{Availability of data and materials}

All data generated or analyzed during this study are included in this published article and its additional files.

\section{Authors' contributions}

AC collected and identified the leaves of Annona purpurea. OE performed the phytochemical study and identified the isolated compounds. CAC conceived and designed the in vitro experiments involving platelet activity. MCR and FM designed and analyzed the results of the calcium experiments. GS, GA and CAC performed the platelet aggregation and secretion experiments and the CAMP measurements. FP, GS, GA and CAC performed the intra-platelet calcium measurements. CAC performed the adhesion experiments. All authors analyzed their respective data. CAC was the major contributor to writing the manuscript and all authors read and approved the final manuscript.

\section{Ethics approval and consent to participate}

The experimental protocol and written informed consent used were approved by the Bioethical Committee of IVIC (number 1316, approval on March 2009), following the guidelines of the Declarations of Helsinki and Tokyo for humans.

\section{Competing interests}

The authors declare that they have no competing interests.

\section{Publisher's Note}

Springer Nature remains neutral with regard to jurisdictional claims in published maps and institutional affiliations.

\section{Author details}

${ }^{1}$ Centro de Biofísica y Bioquímica (CBB), Instituto Venezolano de Investigaciones Científicas (IVIC), Caracas, Bolivarian Republic of Venezuela. ${ }^{2}$ Laboratorio de Botánica Sistemática, Facultad de Agronomía, Universidad Central de Venezuela (UCV), Maracay, Bolivarian Republic of Venezuela. ${ }^{3}$ Laboratorio de Hemostasia y Genética Vascular, Centro de Biofísica y Bioquímica, Instituto Venezolano de Investigaciones Científicas, Apartado 20632, K11 de la Carretera Panamericana, Caracas 1020-A, Bolivarian Republic of Venezuela.

Received: 24 November 2017 Accepted: 9 April 2018

Published online: 18 April 2018

\section{References}

1. Chua D, Nishi C. New antiplatelet agents for cardiovascular disease. Can Med Assoc J. 2013;185:1405-11.

2. Lau WC, Gurbel PA. Antiplatelet drug resistance and drug-drug interactions: role of cytochrome P450 3A4. Pharm Res. 2006;23:2691-708.

3. World Health Organization: The top 10 causes of death. Accessed 4 Apr 2018. http://www.who.int/gho/mortality_burden_ disease/causes_death/top_10/en/

4. El Haouari M, Rosado JA. Medicinal plants with antiplatelet activity. Phytother Res. 2016;30:1059-71.

5. Wu YC, Chang GY, Ko FN, Teng CM. Bioactive constitutents from the stems of Annona montana. Planta Med. 1995; 61:146-9.

6. Chang FR, Wei JL, Teng CM, Wu YC. Antiplatelet aggregation constituents from Annona purpurea. J Nat Prod. 1998;61:1457-61.

7. Chang FR, Wei JL, Teng CM, Wu YC. Two new 7-dehydroaporphine alkaloids and antiplatelet action aporphines from the leaves of Annona purpurea. Phytochemistry. 1998;49:2015-8.

8. $\quad$ Yang YL, Chang FR, Wu CC, Wang WY, Wu YC. New ent-kaurane diterpenoids with anti-platelet aggregation activity from Annona squamosa. J Nat Prod. 2002;65:1462-7. 
9. Missouri Botanical Garden: Tropicos.org. Saint Louis, Missouri. Accessed 21 Nov 2017. http://www.tropicos.org/ Name/1600805

10. Nunn B. Some characteristics of mouse platelet aggregation and a comparison of the activity of a range of compounds in mouse and human platelet-rich plasma in vitro. Thromb Haemost. 1981;45:1-5.

11. Packham MA, Bryant NL, Guccione MA, Kinlough-Rathbone RL, Mustard JF. Effect of the concentration of $\mathrm{Ca}^{2+}$ in the suspending medium on the responses of human and rabbit platelets to aggregating agents. Thromb Haemost. 1989;62:968-76.

12. Packham MA, Rand ML, Kinlough-Rathbone RL. Similarities and differences between rabbit and human platelet characteristics and functions. Comp Biochem Physiol. 1992;103:35-54.

13. Ware J. Dysfunctional platelet membrane receptors: from humans to mice. Thromb Haemost. 2004;92:478-85.

14. The Plant List (2013). Version 1.1. Accessed 21 Nov 2017. http://www.theplantlist.org/tpl1.1/record/kew-2640992

15. Cazenave JP, Ohlmann P, Cassel D, Eckly A, Hechler B, Gachet C. Preparation of washed platelet suspensions from human and rodent blood. Method Mol Biol. 2004;272:13-28.

16. Born G. Aggregation of blood platelet by adenoside diphosphate and its reversal. Nature. 1962;194:927-9.

17. Estrada O, Alvarado-Castillo C, Fernández AZ, López M, Romero-Vecchione E, Vásquez J, Mendez J, Conde D, Cardozo A. Pomolic acid isolated from the leaves of Licania pittieri inhibits ADP-and epinephrine-induced platelet aggregation and has hypotensive effect on rats. Curr Bioact Compd. 2009;5:219-25.

18. Stermitz FR, Castro O. Pentasubstituted aporphine alkaloids from Phoebe molicella. J Nat Prod. 1983;46:913-6.

19. Eriksson AC, Whiss PA. Measurement of adhesion of human platelets in plasma to protein surfaces in microplates. J Pharmacol Toxicol Methods. 2005;52:356-65.

20. Pérez JF, Ruiz MC, Chemello ME, Michelangeli F. Characterization of a membrane calcium pathway induced by rotavirus infection in cultured cells. J Virol. 1999:73:2481-90.

21. Grynkiewicz G, Poenie M, Tsien RY. A new generation of $\mathrm{Ca}^{2+}$ indicators with greatly improved fluorescence properties. J Biol Chem. 1985;260:3440-50.

22. Varga-Szabo D, Braun A, Nieswandt B. Calcium signaling in platelets. J Thromb Haemost. 2009;7:1057-66.

23. Heemskerk JW, Harper MT, Cosemans JM, Poole AW. Unravelling the different functions of protein kinase C isoforms in platelets. FEBS Lett. 2011;585:1711-6.

24. Smolenski A. Novel roles of CAMP/cGMP-dependent signaling in platelets. J Thromb Haemost. 2012;10:167-76.

25. Chia YC, Chang FR, Wu CC, Teng CM, Chen KS, Wu YC. Effect of isoquinoline alkaloids of different structural types on antiplatelet aggregation in vitro. Planta Med. 2006;72:1238-41.

26. Tsuchiya H. Membrane interactions of phytochemicals as their molecular mechanism applicable to the discovery of drug leads from plants. Molecules. 2015;20:18923-66.

27. De La Cruz JP, Arrebola MM, Guerrero A, Sánchez de la Cuesta F. Influence of nitric oxide on the in vitro antiaggregant effect of ticlopidine. Vasc Pharmacol. 2002;38:183-6.

28. Yun SH, Sim EH, Goh RY, Park Jl, Han JY. Platelet activation: the mechanisms and potential biomarkers. Biomed Res Int. 2016;2016:9060143. https://doi.org/10.1155/2016/9060143

29. Menezes LRA, D'Sousa Costa CO, Da C Rodrigues ACB, Do E Santo FR, Nepel A, Dutra LM, Silva FMA, Soares MBP, Barison A, Costa EV, Bezerra DP. Cytotoxic Alkaloids from the Stem of Xylopia laevigata. Molecules. 2016;21:890-900.

30. Bye AP, Unsworth AJ, Gibbins JM. Platelet signaling: a complex interplay between inhibitory and activatory networks. J Thromb Haemost. 2016;14:918-30.

31. Murugappan S, Tuluc F, Dorsam RT, Shankar H, Kunapuli SP. Differential role of protein kinase C $\delta$ isoform in agonist-induced dense granule secretion in human platelets. J Biol Chem. 2004;279:2360-7.

32. Yue C, Dodge KL, Weber G, Sanborn BM. Phosphorylation of serine 1105 by protein kinase A inhibits phospholipase CB3 stimulation by Gaq. J Biol Chem. 1998;273:18023-7.

33. Supattapone S, Danoff SK, Theibert A, Joseph SK, Steiner J, Snyder SH. Cyclic AMP-dependent phosphorylation of a brain inositol trisphosphate receptor decreases its release of calcium. Proc Natl Acad Sci U S A. 1988;85:8747-50.

34. Rondina MT, Weyrich AS. Targeting phosphodiesterases in anti-platelet therapy. Handb Exp Pharmacol. 2012;210:225-38

35. Schwarz UR, Walter U, Eigenthaler M. Taming platelets with cyclic nucleotides. Biochem Pharmacol. 2001:62:1153-61.

36. Hunter RW, Mackintosh C, Hers I. Protein kinase C-mediated phosphorylation and activation of PDE3A regulate cAMP levels in human platelets. J Biol Chem. 2009;284:12339-48.

37. Böhlke M, Guinaudeau H, Angerhofer CK, Wongpanich V, Soejarto DD, Farnsworth NR. Costaricine, a new antiplasmodial bisbenzylisoquinoline alkaloid from Nectandra salicifolia trunk bark. J Nat Prod. 1996;59:576-80. 